Erschienen in: Harras, Gisela (Hrsg.): Die Ordnung der Wörter. Kognitive und lexikalische Strukturen. - Berlin, New York: de Gruyter, 1995. S. 355-358. (Institut für deutsche Sprache. Jahrbuch 1993)

\title{
Podiumsdiskussion: Kognitivismus und Lexikon
}

Für die Podiumsdiskussion stand ein ganzer Nachmittag der Tagung zur Verfügung. Die Diskutanten waren: Roland Mangold-Allwinn, Manfred Bierwisch, Monika Schwarz, Manfred Wettler und Gisela Harras (Moderation). Diskussionsschwerpunkte waren: Kognitivismus, kognitive Wissenschaften und Linguistik; der Status "mentaler Repräsentationen”; die Unterscheidung zwischen konzeptueller und semantischer Ebene sowie die Frage, was sich unter kognitivistischen Aspekten an der linguistischen (Wort)Semantik ändern kann oder sollte. Nachfolgend sind die Statements zweier Diskutanten abgedruckt. Leider lagen bei Redaktionsschluß nur diese beiden Manuskripte vor.

\section{ROLAND MANGOLD-ALLWINN}

\section{Kognitivismus, kognitive Wissenschaft und Linguistik}

Außer in der Linguistik und Psycholinguistik wird auch in der Sprachund Kognitionspsychologie die Sprachverarbeitung beim Menschen untersucht. Dabei wird seit der kognitiven Wende (s.u.) von der Mehrzahl der Kognitionspsychologen folgende Auffassung vom Sprechen und Sprachverstehen vertreten: Ein sprachlicher Input wird vom kognitiven System "Mensch" aufbereitet und führt zu bestimmten internen Repräsentationen (vgl. Herrmann (1985); Levelt (1989)). (Wie diese Repräsentationen beschrieben werden können, wird in Punkt 2 diskutiert.) Andererseits gehen bei der Sprachproduktion sprachliche Outputs aus internen Repräsentationen hervor. Dabei wird in der neueren Kognitionspsychologie überwiegend ein Ansatz verfolgt, wonach sprachliche Anteile ("Wortmarken" bei Herrmann (1985) und Engelkamp (1990)) von nichtsprachlich-semantischen Anteilen („Nichtwort-Marken”) solcher Repräsentationen unterschieden werden. (Beispielsweise sind alle Informationen über das Wort "Apfel” den Wort-Marken zuzurechnen, während das Wissen über den Begriff APFEL den eigentlich semantischen Marken zugehört.)

Marken sind beim Menschen in symbolischer Form repräsentiert, was zur Folge hat, daß interne Zustände interpretierbar sind. In einer Reihe von Ansätzen wird diesen Symbolen ein variabler Aktivationszustand zugewiesen, d.h., je nach dem Betrag dieser Aktivation steht das Symbol bei der ablaufenden Informationsverarbeitung mehr oder weniger im Vordergrund. Im Rahmen der konnektionistischen Modellierung, deren Bedeutung für die Kognitionspsychologie zunehmend erkannt wird, wird versucht, durch Erhöhung der Knotenzahl mit gleichzeitiger verteil- 
ter Kodierung auf symbolisch interpretierbare Knoten zu verzichten und alleine mit Aktivationsmustern als Grundlage aller Sprachverarbeitung auszukommen. (vgl. Mangold-Allwinn (1993)).

Ohne langzeitlich überdauerndes Wissen wäre Sprechen und Sprachverstehen beim Menschen nicht möglich. Es wird in der Kognitionspsychologie $\mathbf{z}$ wischen diesem - potentiell nutzbaren - Wissen im Langzeitgedächtnis und der im Arbeitsgedächtnis repräsentierten aktuellen Information unterschieden. Auch Langzeitwissen umfaßt die bereits diskutierten sowohl sprachlichen (z.B. syntaktische Regeln, Lexikon) als auch nichtsprachlichen Informationen (z.B. daß ein Pappbecher ein Picknickutensil ist).

\section{Wie realistisch ist die Annahme ,mentaler Repräsentationen"?}

Zur Zeit des Behaviorismus galten kognitive Vorgänge beim Menschen als nicht objektiv untersuchbar und wurden von der Forschung ausgeschlossen. (Erste Aufweichungen waren im Neobehaviorismus erkennbar, indem von prominenten Vertretern interne bzw. vermittelnde Reaktionen als wichtige Elemente ihrer Theorien postuliert wurden.) Nach der kognitiven Wende richtete sich die Aufmerksamkeit der kognitiven Psychologen dann intensiv auf das Studium der Organisation mentaler Repräsentationen und deren Nutzung bei der Informationsverarbeitung. Insofern ist es für einen Kognitionspsychologen selbstverständlich, mentale Repräsentationen als Bestandteile seiner Modellierungen vorzusehen. (Allerdings besteht Uneinigkeit über den Status, der diesen mentalen Repräsentationen bei der Erklärung zukommt; vgl. Engelkamp/Pechmann (1988) sowie die Antwort von Herrmann (1988).)

Auf die Unterscheidung von eher nichtsprachlichen und von eher sprachlichen Anteilen mentaler Repräsentationen - insbesondere bei der Erforschung von Vorgängen beim Sprechen und Sprachverstehen - wurde bereits in der Stellungnahme zum ersten Punkt hingewiesen. Von Autoren wie Herrmann (1985) oder Engelkamp (1990) wird eine weitere Unterteilung der zur Repräsentation angenommenen Marken vorgenommen. So findet man in der Mannheimer Arbeitsgruppe um Herrmann einen Ansatz modalitätsspezifischer Marken, d.h., es werden imaginale (perzeptnahe), motorische sowie abstrakt-semantische Marken angenommen. Beispielsweise ist die imaginale Nichtwort-Marke eines Apfels das Vorstellungsbild dieses Apfels (seine Form, seine Farbe, sein Duft), und die motorische Nichtwort-Marke beinhaltet etwa ein Handlungsprogramm für das Pflücken eines Apfels. Die semantisch-abstrakte Nichtwort-Marke eines Apfels wiederum enthält Information dazu, daß ein Apfel zu den 
Früchten gerechnet wird. Die imaginale Wort-Marke umfaßt Information zum Aussehen des geschriebenen Wortes „Apfel” bzw. zum Klang des gesprochenen Wortes. Die motorische Marke ermöglicht ein Schreiben bzw. Aussprechen dieses Wortes. SchlieBlich beinhaltet die abstraktsemantische Wort-Marke Informationen darüber, daß das Wort „Apfel” ein Substantiv ist und wie es dekliniert wird.

Bei der Verarbeitung liegen die zum Objekt „Apfel” gehörenden Marken in unterschiedlicher Aktivationsstärke im Arbeitsgedächtnis vor. Die Bennenung eines A pfels kann so modelliert werden, daB - über das Wahrnehmungssystem - zunächst die imaginalen Marken dominant aktiviert werden und daß über Verbindungen zwischen den Marken im Verlauf der Aktivationsausbreitung auch die motorische Wort-Marke aktiviert werden. Die Ausführung des Handlungsprogrammes bringt schließlich den sprachlichen Output.

3. In welcher Weise sind konzeptuelle und semantische Ebene aufeinander bezogen?

Es wurde aus den Ausführungen zu Punkt 1 und 2 bereits erkennbar, daB in der Kognitionspsychologie nicht die Trennung in konzeptuelle und semantische Ebene im Vordergrund steht. Überhaupt tritt aus meiner Sicht die Konzeption getrennter Ebenen, d.h. die Annahme unabhängig voneinander arbeitender Teilsysteme, zugunsten der Annahme einer mehr parallelen und integrierten Verarbeitung unterschiedlicher Repräsentationsarten in den Hintergrund. Als Beleg für diese These kann die Modellierung einer gleichzeitigen Verarbeitung von sowohl nichtsprachlichen als auch sprachlichen Marken im Mannheimer Ansatz (vgl. Herrmann (1985)) gelten.

4. Was ändert sich in der Linguistik, wenn die (Wort-) Semantik ,kognitiv" wird?

Es wurde bereits erläutert, daß aus der Sicht eines Kognitionspsychologen eine andere als „kognitive" Auffassung vom menschlichen Sprechen und Sprachverstehen gar nicht vorstellbar ist. Wenn sich die Linguistik als eine Wissenschaft auch vom Menschen begreift, die ihre Aufgabe nicht lediglich darin sehen will, sprachliche Inputs bzw. Outputs in ihrer Beschaffenheit detailliert zu beschreiben und zu klassifizieren, wird sie um eine - in der Psychologie bereits vollzogene - kognitive Wende nicht herumkommen. Damit wäre aber die Frage einer Grenzziehung zwischen solchen Disziplinen wie der Linguistik, der Psycholinguistik und der kognitiven Psychologie angesprochen. 


\section{Literatur}

Engelkamp, Johannes (1990): Das menschliche Gedächtnis: das Erinnern von Sprache, Bildern und Handlungen. Göttingen/Hogrefe.

Engelkamp, Johannes/Pechmann, Thomas (1988): Kritische Anmerkungen zum Begriff der mentalen Repräsentation. In: Sprache \& Kognition 7, S. 2-11.

Herrmann, Theo (1985): Allgemeine Sprachpsychologie. München. Urban \& Schwarzenberg.

Herrmann, Theo (1988): Mentale Repräsentation - ein erlāuterungsbedürftiger Begriff. In: Sprache \& Kognition 7, S. 162-175.

Levelt, Willem J.M. (1989): Speaking. From intention to articulation. Cambridge, Mass: MIT Press.

Mangold-Allwinn, Roland (1993): Flexible Konzepte: Experimente, Modelle, Simulationen. Frankfurt: Lang. 